\title{
La importancia del estudio de los clásicos en la formación académica*
}

\section{Corrado Petrocelli}

corrado.petrocelli@uniba.it

Università di Bari

Resumen: El presente trabajo pretende rescatar la importancia del estudio de los clásicos con la finalidad de reconocer, recobrar y revalorar su repercusión en la formación académica. En un primer momento se analiza la cultura greco-romana y se reflexiona sobre la herencia dejada por los antiguos como fundamento del pensamiento filosófico moderno. En un segundo momento nos detenemos a analizar el trabajo de los Padres de la Iglesia, quienes revaloraron el legado del mundo antiguo, en particular, la literatura, para repotenciar la formación cristiana de los jóvenes. En un tercer momento se analiza el fenómeno de la «traducción de la Biblia», cuya repercusión transformó de manera trascendental la cultura, se reconoce a la «traducción» como un proceso de humanización del hombre que consolida la cultura humanística. En este sentido, se reflexiona sobre la conciencia de la alteridad presente en los clásicos. Finalmente, se concluye argumentando que (a) el estudio de los clásicos educa a los hombres en el arduo trabajo de desarrollar un pensamiento crítico, y (b) las generaciones que se encuentren con este legado reconozcan en ellos la fuente de inspiración que les permita hurgar en su interior y repotenciar una nueva forma de vida.

Palabras clave: Clásicos, cultura greco-latina, pensamiento filosófico, formación cristiana, traducción de la Biblia, cultura humanística, alteridad, educar a los jóvenes.

*Traducción de Seleni Adilia Díaz Vargas. 


\title{
The importance of studying classical scholars in the academic
} formation

\begin{abstract}
The current research seeks to rescue the importance of the classics study aiming at recognizing, recovering, and revaluing its influence in academic development. First, an analysis is made on the Greek-roman culture and then reflection is carried out on it as the foundations for the modern philosophical thought. Second, the Church Masters' work is analyzed since they revalued the legacy of the ancient world, particularly, its literature, in order to reorient the Christian formation of the youth. Third, the «Bible Translation» phenomenon is worked out, since its repercussion transcendentally transformed the culture. «Translation» is recognized as man's humanization process that consolidates the humanistic culture. From this posture, a reflection on the «otherness», which is present in the classics, is made. Finally, the work is concluded by making the following arguments: (a) the study of the classics educates man in critical-thinking development; (b) the generations that are taught this legacy acknowledge it as a source of inspiration that allows them to rummage inside and reinstate a new form of life.
\end{abstract}

Keywords: Classics, Greek-Latin culture, philosophical thought, Christian formation, Bible translation, humanistic culture, «otherness», youth education.

Criederich Hegel solía dar inicio a sus clases de Historia de la Filosofía —en los cursos afirmación que se convirtió rápidamente en una frase célebre: «Al nome Grecia l'uomo colto [...] si sente a proprio agio, come se fosse a casa propia» (1930: p. 167). La razón — prosigue Hegel— es que la religión y el más allá provienen del Oriente.

Ma il qui, il presente, la scienza e l'arte, tutto ciò che, mentre soddisfa il nostro spirito, gli conferisce dignità e ornamento, noi sappiamo che ci è venuto dalla Grecia o direttamente o indirettamente per il tramite dei Romani. Quest'ultima via rappresenta la prima forma, in cui la civiltà greca pervenne a noi, anche per opera della Chiesa già universale, che come tale trae la sua origine da Roma e ha 


\section{LA IMPORTANCIA DEL ESTUDIO DE LOS CLÁSICOS EN LA FORMACIÓN ACADÉMICA}

conservato fino a oggi perfino la lingua dei Romani. Le fonti dell'insegnamento, accanto al Vangelo latino, sono stati i Padri della Chiesa. $(1930: 167)^{1}$

El pensamiento filosófico moderno tiene su propio origen en el mundo antiguo. Asimismo, reconoce que de aquel mundo, de sus sistemas, de sus conceptos e incluso de su lenguaje se introdujeron los elementos constitutivos de su propia estructura y de su propia evolución.

Los antiguos quisieron conocer la naturaleza a través de la especulación y dominarla con la técnica. Toda la historia de la cultura clásica, sobre todo helénica, se afirma como un constante emerger desde lo fantástico hasta lo racional, del mito al logos. El logos se concibe como raciocinio, palabra, discurso, y logos como razonamiento y relación dinámica, abierta a la dialéctica y al encuentro con la experiencia y las culturas ajenas. Un breve texto de Jorge Luis Borges, titulado El principio, explica la medida en que los griegos lograron liberarse de la penumbra de la superstición.

Due greci stanno conversando: Socrate e Parmenide, forse. [...] Il tema del dialogo è astratto. Talora alludono a miti nei quali entrambi non credono. Le ragioni che adducono possono abbondare in errori e non hanno uno scopo. Non polemizzano e non vogliono né persuadere né essere persuasi, non pensano né a vincere né a perdere. Sono d'accordo su una cosa sola: sanno che la discussione è la non impossibile via per giungere a una verità. Liberi dal mito e dalla metafora, pensano o cercano di pensare. Non sapremo mai i loro nomi. Questa conversazione fra due sconosciuti in un luogo della Grecia è il fatto capitale della storia. Hanno dimenticato la preghiera e la magia. ${ }^{2}$ (1985: 1341)

1 «Pero las concepciones del más acá, de lo presente, la ciencia y el arte, lo que satisface, dignifica y adorna nuestra vida espiritual, tuvo como punto de partida a Grecia, bien directamente, bien indirectamente, a través de los romanos. Este último camino, el de Roma, fue la primera forma en que esta cultura llegó a nosotros, por parte también de la Iglesia, en otro tiempo universal, cuyo origen debe buscarse en la misma Roma y que todavía hoy conserva la lengua de los romanos. Las fuentes de la enseñanza eran el Evangelio latino y los Padres de la Iglesia» (p. 139).

2 «Dos griegos están conversando: Sócrates acaso y Parménides.

Conviene que no sepamos nunca sus nombres; la historia, así, será más misteriosa y más tranquila.

El tema del diálogo es abstracto. Aluden a veces a mitos, de los que ambos descreen.

Las razones que alegan pueden abundar en falacias y no dan con un fin.

No polemizan. Y no quieren persuadir ni ser persuadidos, no piensan en ganar o en perder.

Están de acuerdo en una sola cosa; saben que la discusión es el no imposible camino para llegar a una verdad. Libres del mito y de la metáfora, piensan o tratan de pensar.

No sabremos nunca sus nombres.

Esta conversación de dos desconocidos en un lugar de Grecia es el hecho capital de la Historia. 
El hombre griego no ignora ni los fantasmas de la imaginación, ni las zonas oscuras de la existencia, ni las flechas inesperadas del destino; sin embargo, su mente es llevada a ver claro, dentro y fuera de sí. En los enredos del mundo de la experiencia, el hombre griego tiende a poner orden y método; donde el transcurso de los acontecimientos aparece desconectado y convulso, sabe encontrar un ritmo que él mismo aprende después a medir. Donde la disonancia parece reinar soberana, percibe la presencia de una armonía escondida, como se evidencia en la siguiente cita: «Il mondo è kosmos, struttura unitaria e ordinata, che il pensiero è chiamato a riconoscere, a riivelare e a esprimere in numeri, in formole, in figure geometriche, in rapporti quantitativi» (Faggin 1964: 11); ${ }^{3}$ si le fuera posible al hombre también dominaría el cosmos.

Nuestra concepción del mundo se ha hecho más fluida y dinámica y ha colocado al pensamiento aprehensivo al interior de un sistema de relaciones complejas y móviles. Sin embargo, la exigencia de reducir la complejidad a fenómenos específicos, cualificados, mensurables; junto a la plena confianza en la transparente inteligibilidad del ser, y, por el contrario, la desconfianza hacia cuanto no puede ser conducido a las coordenadas de un saber dominado y codificado son aspectos que sustentan el pensamiento moderno. A la luz de tal herencia, que podríamos definir de naturaleza gnoseológica, la actualidad de lo antiguo deviene en un paradigma que traspasa los límites de la aparente paradoja lingüística: esto es posible solo gracias al estudio de los clásicos.

Antonio Gramsci reflexionaba sobre los riesgos que el decaimiento de la instrucción escolástica de sus tiempos habría comportado para la formación de los jóvenes, si no se hubiese afirmado la necesidad de un estudio crítico y consciente de las lenguas clásicas: «Non si imparava il latino e il greco per parlarli, per fare i camerieri, gli interpreti, i corrispondenti commerciali. Si imparava per conoscere direttamente la civiltà dei due popoli, presupposto necessario della civiltà moderna, cioè per essere se stessi e conoscere se stessi consapevolmente» (Gramsci 1975: 1544). ${ }^{4}$

Hablamos de identidad lingüística si pensamos que el latín ha sido la lengua de la ciencia, de la política y de la religión hasta casi todo el siglo xx. Es decir, se trata de una

Han olvidado la plegaria y la magia». (Borges 1990: 415)

3 «El mundo es cosmos, estructura unitaria y ordenada, que el pensamiento es llamado a reconocer, a revelar y a expresar en números, en fórmulas, en figuras geométricas, en relaciones cuantitativas».

4 «No se aprendía el latín y el griego para hablarlo, para ser camareros, intérpretes, corresponsales mercantiles. Se aprendía para conocer directamente la civilización de dos pueblos, presupuesto necesario de la civilización moderna, es decir, para ser uno mismo y conocerse a sí mismo conscientemente». 
identidad en el origen del pensamiento jurídico moderno o en la elaboración de un método científico, de una forma mental abierta a las posibles alteridades, en cuanto heredera de una pluralidad de concepciones del mundo, no siempre congruentes o conciliables.

La consciencia de esta identidad puede traer ejemplos del fermento dialéctico con los cuales los Padres de la Iglesia vivieron las relaciones con la cultura pagana clásica, griega y latina. Esta cultura la quisieron conocer primero antes que condenar, y de la cual declararon obtener elementos de fecunda validez.

Pensemos en la Oratio ad adolescentes de Basilio de Cesarea. En este, dirigiéndose a las generaciones venideras, Basilio exalta los beneficios que la literatura profana ha ejercido para la formación cristiana hasta el punto de considerar a los clásicos un 'viático' para la instrucción y la paideia del joven cristiano, de la cual sacaba provecho para el alma.

Basilio celebra los valores de la literatura pagana en un pequeño tratado protrettico de clara influencia plutarca, en el cual Platón y Plutarco son los clásicos de referencia. Sabe cuánto la propia naturaleza del cristiano está empapada de aquel espíritu que ya a los orígenes tanta parte tuvo en el proceso de formación de la conciencia de la nueva religión, junto a las matrices judaicas del cercano Oriente.

Pensemos en la traducción de la Biblia que los Setenta adentraron en el Egipto de los Tolomeos: las palabras, los temas, los símbolos de la fe israelita fueron traspasados no solo a las formas de la lengua griega, sino también a las categorías culturales y conceptuales griegas, en una versión que hasta su nacimiento asumió la misma autorizada sacralidad del correspondiente texto traducido. Se trató, como advierte Elías Bickerman, de un ejemplo magistral de «ósmosis en las dos direcciones», que fue central no solo en la vicisitud del mundo que definimos, en modo sincrético, judáico-helenístico, sino también en la civilización mundial, como se constata en la siguiente afirmación: «[La traduzione greca dei Settanta] aprì la Bibbia al mondo e il mondo alla parola di Dio. Senza questa traduzione Londra e Roma sarebbero ancora pagane e le Scritture non sarebbero note meglio del Libro dei Morti egiziano» (Bickerman 1991: 145). ${ }^{5}$

La cultura humanística interpreta y decodifica tal identidad, y lo hace en más direcciones. Estas son las siguientes: (a) el valor de la continuidad de la evolución

5 «[La traducción griega de los Setenta] abrió la Biblia al mundo y el mundo a la palabra de Dios. Sin esta traducción Londres y Roma serían aún paganas y las Escrituras no serían conocidas mejor que el Libro de los muertos egipcio". 
histórica; (b) la exigencia de una visión unitaria de la realidad, incluso en la multiplicidad de sus manifestaciones; (c) la dimensión racional de la búsqueda infinita; (d) el momento de la especulación desinteresada; (e) la integridad del ser humano y el sentido de sus límites y de su dignidad; (f) la naturaleza como cosmos. Pero ya que estos valores han tomado forma y expresión en obras artísticas o literarias, la cultura humanística se las ingenia para perpetuar el conocimiento de estos productos de generación en generación, no para difundir un patrimonio ya adquirido de conocimientos, sino para conservar y quizá potenciar un estilo de vida, un modo de mirar la realidad, una categoría del espíritu.

Tal conocimiento profundo del pasado, que presupone una confrontación constante con el presente, no se puede emprender de manera correcta, sino a través del instrumento de la traducción. Nos referimos a un proceso mental de tipo intuitivo-reconstructivo, en el cual interactúan de una parte la intuición —aquella del significado general del texto- y de la otra la interpretación, de las palabras individuales y de cada frase. La validez de este proceso mental se explica también en otros campos, aparentemente del todo ajenos a la cultura humanística: de este modo se educan las mentes para el juicio, para la crítica, para la creatividad. En 1869 Giuseppe Müller, docente de griego en Turín, escribía en un breve prefacio a la versión italiana de los Ejercicios Griegos de Karl Schenkl:

Le scuole classiche si propongono educare uomini, o, in altri termini, avvezzare i giovani alla difficile opera del pensiero e al pieno e naturale uso delle loro forze intellettive. Le dottrine linguistiche, come quelle che s'occupano dell'organo pel cui mezzo formuliamo il pensiero, per ciò che pensare è parlare, manifestamente sono le meglio atte ad educare le facoltà intellettive, e l'esperienza di molti secoli conferma essere lo studio delle lingue il mezzo più certo per risvegliarle [...]. Studiare la lingua d'un popolo val quanto vivere la vita di questo popolo, per ciò che sia appropriarsi il suo modo di pensare; ed è ormai ammesso universalmente, che tra il modo di pensare e d'esprimersi e tutte le altre manifestazioni della vita d'un popolo sia relazione strettissima. ${ }^{6}$ (Müller 1869, pp. III-Iv)

6 «Las escuelas clásicas se proponen educar a los hombres, o, en otros términos, acostumbrar a los jóvenes al difícil trabajo del pensamiento y al pleno y natural uso de sus fuerzas intelectuales. Las doctrinas lingüísticas, como aquellas que se ocupan del órgano por cuyo medio formulamos el pensamiento, por eso es que pensar es hablar, manifiestamente son las más aptas para educar las facultades intelectuales, y la experiencia de muchos siglos confirma ser el estudio de las lenguas el medio más cierto para despertarlas [...]. Estudiar la lengua de un pueblo vale cuanto vivir la vida de este pueblo, por el hecho de que es apropiarse de su modo de pensar; y es ya admitido universalmente que entre el modo de pensar y el de expresarse y todas las otras manifestaciones de la vida de un pueblo haya una relación estrechísima». 
El latín — así reflexiona Müller—es la lengua del intelecto por excelencia, el griego del arte y de la belleza, a la cual se necesita conducir a aquellos jóvenes que quieren consagrarse no tanto a los estudios clásicos, cuanto «a los buenos estudios, a las nobles artes, a la ciencia y a los magisterios más solemnes de la vida social». Por esto, el latín y el griego representan el mejor instrumento para «educar la mente y formar al hombre», allá donde es evidente el fracaso de muchas tentativas de sustituir a la enseñanza de las lenguas clásicas otras materias de estudio, consideradas más útiles para la vida práctica. Pero, de esta manera — concluye Müller— arrebatadas las lenguas clásicas de la escuela, «vengono a mancare le più solide fondamenta all'educazione giovanile» (Müller 1869: V-VII).$^{7}$

A tal propósito, escribe Martha Nussbaum en una reciente y valorada contribución:

La spinta al profitto induce molti leader a pensare che la scienza e la tecnologia siano di cruciale importanza per il futuro dei loro paesi. [...] La mia preoccupazione è che altre capacità, altrettanto importanti, stiano correndo il rischio di sparire nel vortice della concorrenza: capacità essenziali per la salute di qualsiasi democrazia e per la creazione di una cultura mondiale in grado di affrontare con competenza i più urgenti problemi del pianeta. Tali capacità sono associate agli studi umanistici e artistici: la capacità di pensare criticamente, la capacità di trascendere i localismi e di affrontare i problemi mondiali come «cittadini del mondo»; e, infine, la capacità di raffigurarsi simpateticamente la categoria dell'altro. ${ }^{8}$ (Nussbaum 2011: 26)

El interés de los países modernos por aquellas competencias que produzcan una ventaja a corto plazo, reduce el valor de la importancia de otras capacidades como la crítica a la tradición y a la autoridad, el análisis de sí mismo y del otro, el análisis de la razón; capacidades que solo la cultura humanística puede suscitar y cultivar, a través de la filosofía, la historia, la literatura y el arte. Los medios de comunicación moderna — subraya Nussbaum— aman las frases lapidarias

«Vienen a faltar los más sólidos fundamentos de la educación juvenil».

8 «El impulso hacia el beneficio induce a muchos líderes a pensar que la ciencia y la tecnología son de crucial importancia para el fututo de sus países. [...] Mi preocupación es que otras capacidades, igualmente importantes, están corriendo el riesgo de desaparecer en el torbellino de la concurrencia: capacidades esenciales para la salud de cualquier democracia y para la creación de una cultura mundial capaz de afrontar con competencia los problemas más urgentes del planeta. Tales capacidades son asociadas a los estudios humanísticos y artísticos: la capacidad de pensar críticamente, la capacidad de trascender los localismos y de afrontar los problemas mundiales como "ciudadanos del mundo»; y, en fin, la capacidad de representarse empáticamente la categoría del otro». 
y sustituyen la discusión con la acusación, dando origen a una política degradada. A través de la filosofía, en cambio, los jóvenes aprenden a desarrollar el argumento del adversario, y a descubrir los elementos comunes y las divergencias. Al lado de la filosofía, la historia, la historia del mundo y de las religiones, el estudio comparado de las culturas se convierten en esenciales para favorecer una discusión crítica sobre los problemas del mundo, poniendo las premisas para aquel esfuerzo llamado positional thinking, de 'imaginación cultivada', que es el pensar desde el punto de vista del otro. Y es por eso que tenemos necesidad de la literatura y del arte, a través de las cuales aprender a ver a otros diversos a nosotros, no solo como «el otro» amenazador, sino como seres humanos iguales, aunque con aspiraciones y objetivos propios.

A la luz de tales consideraciones, el camino más correcto para interpretar lo antiguo, y para vivir y pensar el presente a la luz de las enseñanzas de los antiguos, está precisamente en la conciencia de la alteridad y en el conocimiento per differentiam, es decir, conocer al otro en su diversidad. Los clásicos nos interesan, sostiene Eduardo Sanguineti "perché sono da noi radicalmente diversi. Sono radicalmente esotici, [...] temporalmente come spazialmente, almeno per metafora. Importano perché additano forme di esperienza da noi remote, anche impraticabili, e anche, non di rado, incomprensibili, ma che, appunto per questo, ci aprono a dimensioni diverse, altrimenti ignote e insospettabili» (Sanguineti 2002: 211). ${ }^{9}$

Tal dialéctica vuelve también comprensible la permanente actualidad del mundo antiguo quod ad nos - lo que es para nosotros- por como nosotros mismos lo entendemos. Por eso, puede aún hoy recibir estatuto de validez el conocido aforismo medieval sobre los enanos y los gigantes:

Bernardo di Chartres diceva che noi siamo come nani che stanno sulle spalle dei giganti, così che possiamo vedere più lontano di loro non a causa della nostra statura o dell'acutezza della nostra vista, ma perché — stando sulle loro spalle— stiamo più in alto di loro. ${ }^{10}$ (Jeauneau 1969)

9 «Porque son radicalmente diversos a nosotros. Son radicalmente exóticos, temporalmente como espacialmente, al menos como metáfora. Importan porque adhieren formas de experiencias remotas a nosotros, también impracticables, y también, no raras veces, incomprensibles, pero que, precisamente por esto, nos abren a dimensiones diversas, en otros casos ignotas e insospechables».

10 «Bernardo di Chartres decía que nosotros somos como enanos que están sobre la espalda de los gigantes, así que podemos ver más lejos que ellos no a causa de nuestra estatura o de la agudeza de nuestra vista, sino porque estando sobre sus espaldas estamos más alto que ellos». 


\section{LA IMPORTANCIA DEL ESTUDIO DE LOS CLÁSICOS EN LA FORMACIÓN ACADÉMICA}

El aforismo es atribuido a Bernardo di Chartres de Giovanni di Salisbury en el Metalogicon. Estamos en el siglo xir. Después del Metalogicon se registra una serie de ocurrencias, en una colección de sentencias teológicas ligadas a la escuela de Laon, ${ }^{11}$ en Gerardo de Cambrai, ${ }^{12}$ en el histórico danés Sven Aggense, ${ }^{13}$ en Gerardo de Alvernia, ${ }^{14}$ en Alexander Ricart, médico en la corte de Aragón entre 1395 y 1422, ${ }^{15}$ hasta Gassendi ${ }^{16}$ y, para llegar a nuestros tiempos próximos, también en Ortega y Gasset; hasta una citación de Max Gluckman en Jeremy Rifkin, según el cual: «scienza è qualsiasi disciplina in cui anche uno stupido di questa generazione può oltrepassare il punto raggiunto da un genio della generazione precedente». ${ }^{17}$

11 Ipsa vero divina pagina quasi quidam altissimus gigas est, nos vero quasi parvuli pueri. Opportet ergo ut humiliet se et condescendat nobis ipsa divina scriptura, taliter loquens ut eam intelligere possimus. Ita plane facit. Condescendit nobis ipse altissimus gigas ita ut super eum ascendere possimus. Cumque super eum ascenderimus, elevat nos usque ad nubes (Rouen, Bibl. mun. 553, fol. 134v).

12 Hoc etiam animositatis tam scintillam adiicere quam et innatum ignem inflammare potest, quod tantis auctoribus et tot innitentes, eorumque tanquam humeris insidentes, multiplicataeque maiestatis beneficio magni fieri possimus, si magnanimi (Topographia Hibernica, Introitus, in: E. Jeauneau, Nani sulle spalle di giganti, Napoli 1969, p. 50).

13 H>actenus, quantum a veteribus et annosis inquisitione diligenti poteram investigare, oratione licet incontexta legem castrensem explicui. Superest ut posteri nostri, quos super humeros gigantum nanos esse autumat autoritas, $<$ ista> verborum scematibus orationeque perornent falerata, tractatus subplendo defectum stylo consummantes elegantiori (Lex Castrensis sive Curiae, Epilogus: ibid., p. 50).

14 Quasi ego nanus super humeros gygantum positus, eorum beneficio speculabor longius quam et ipsi; elegantiores eorum sentencias, quas jam vetustas hominumve neglectus quasi jam mortuas aboleverat, in quandam novitatem essenciae suscitavi (L. Delisle, Le chroniqueur Girard d'Auvergne ou d'Anvers, "Journal des savants», 1900, p. 235).

15 Non est quo mireris ex eadem materia suisque studiis diversos diversa apta colligere; in eodem prato bos herbam quaerit, canis leporem, ciconia lacertam. Fateor tamen quod sum, comparatus ad illos, sicut Minalio pygmaeus in collo Atlantis erectus (Préface adressée au roi Martin: ibid., p. 56).

16 Hinc si existimemus naturam non iam homines gignere, sed absurdos tantum simias: si nos ut nanos despiciamus, suscipiamusque veteres quasi aliquos gigantes: ita quidem esse continget: aut iniqua potius damnatione nostri quam ullo naturae vitio. Illa quippe non minus in nos quam in illos liberalis fuit, si modo sedulitate atque diligentia velimus contendere: si expendamus attentius non tantum quid ferre recusent, sed et quid valeant humeri. Revera enim, si ut antiqui animum applicaremus, eveheremur longe altius: illorumque adiuti subsidiis in giganteam quandam molem excresceremus tandem aliquando (Exercitations paradoxicae adversus Aristoteleos I, 2.13).

17 «Ciencia es cualquier disciplina en la cual también un estúpido de esta generación puede superar el punto alcanzado por un genio de la generación precedente». 
En los ocho siglos que transcurren entre Giovanni de Salisbury y Max Gluckman el aforismo ha sufrido una transformación conceptual que puede representar una señal significativa de la relación que las generaciones instauran con las precedentes. Bernardo de Chartres intervenía en el debate surgido en el ámbito retórico acerca del concepto de conocimiento y de imitación de los antiguos: no se trataba, según su punto de vista, de imitar acríticamente el estilo de los antepasados, sino de aprender de ellos a escribir bien, con la finalidad de ser inspiradores de las generaciones sucesivas de escritores, tanto cuanto lo habían sido aquellos autores de quienes se ha aprendido (Cf. Eco 2002: 115-139).

Esta apelación a la alteridad consciente en el estilo ornamentado se convierte en afirmación que en nuestros días connota el carácter progresivo de la ciencia en el signo de un diálogo crítico y vital con el pasado y de las expectativas que el presente proyecta en el futuro. Se está ante una confrontación cerrada que es necesaria emprender siempre en función del presente. Lo explica naturalmente una consideración de Nietzsche, cuyo espíritu, a través de la cita de Benedetto Croce, ha sido asumida come epígrafe de «La palabra del pasado», una revista italiana de estudio de la antigüedad: «La parola del passato è sempre simile a una sentenza d'oracolo, e voi non la intenderete se non in quanto sarete gli intenditori del presente, e gli artefici dell'avvenire». ${ }^{18}$

En la República, Cicerón hace de Scipione, el hombre magnánimo que podrá salvar a Roma mostrando a la patria la luz del ingenio y de la sabiduría (lumen animi, ingenii consiliique: República. vi,12), solo después de haber admirado el cosmos y escuchado la armonía de las esferas celestes, aquel sonido que «legato a intervalli di natura diversa, ma tuttavia distinti in proporzione secondo un principio tradizionale, è prodotto dalla spinta e dal movimento delle orbite stesse, e che, temperando le note acute con le gravi, genera melodie armoniosamente varie» (República vi,18). ${ }^{19}$ En el Primer discurso inaugural a la presidencia de los Estados Unidos del 4 de marzo de 1861, Abraham Lincoln evitaba la amenaza de guerra civil inminente sobre el Estado, recién constituido, mediante una metáfora coherente con el lugar ciceroniano, metáfora que aún hoy pertenece al léxico de la oratoria política americana. Lincoln habló de los acordes místicos de la memoria (mystic

18 «La palabra del pasado es siempre igual a una sentencia del oráculo y no la comprenderán si no en cuanto hayan comprendido el presente y sean los artífices del futuro».

19 Ille qui intervallis coniunctus inparibus, sed tamen pro rata parte ratione distinctis, inpulsu et motu ipsorum orbium efficitur, et acuta cum gravibus temperans varios aequabiliter concentus efficit (trad. F. Nenci). 
chords of memory), que partiendo de cada campo de batalla, «avrebbero invaso la vastità delle terre appena conquistate e alimentato il coro dell'unione quando suonate dai migliori angeli della nostra natura». ${ }^{20}$

Martin Luther King escogió el monumento de Abraham Lincoln en Washington para dar su célebre discurso I have a dream: aquí evocó aquellos 'acordes de la memoria' y, con ellos, la promesa del pasado, que los Padres de la Constitución, los 'arquitectos de la república' (architechts of our republic) habían firmado: la promesa que todos los hombres hubieran ahora y por siempre gozado del derecho inalienable a la vida, a la libertad, a la búsqueda de la felicidad. ${ }^{21}$ Palabras densas e importantes para la democracia y la libertad más allá de los pueblos de occidente; no es casualidad que fueran pronunciadas junto al monumento de Abraham Lincoln: un gran templo en estilo griego.

20 The mystic chords of memory, stretching from every battlefield and patriot grave to every living heart and hearthstone all over this broad land, will yet swell the chorus of the Union, when again touched, as surely they will be, by the better angels of our nature.

${ }_{21}$ In a sense we have come to our nation's capital to cash a check. When the architects of our republic wrote the magnificent words of the Constitution and the Declaration of Independence, they were signing a promissory note to which every American was to fall heir. This note was a promise that all men would be guaranteed the inalienable rights of life, liberty, and the pursuit of happiness. 


\section{REFERENCIAS}

BORGES, Jorge Luis

«Il principio». En Borges, Jorge Luis, Tutte le opere, a cura di D. Porzio, vol. II. Milano: Mondatori.

1990 Obras Completas. 18. ${ }^{a}$ ed. Tomo III. Buenos Aires: Emecé Editores.

Bickerman, Elías Joseph

1991

Gli Ebrei in età greca. Bologna: Il Mulino.

Canfora, Luciano

2002

Noi e gli antichi. Perché lo studio dei Greci e dei Romani giova all'intelligenza dei moderni. Milano: Rizzoli.

2012

Disegnare il futuro con intelligenza antica. Linsegnamento del latino e del greco antico in Italia e nel mondo. A.c. di U. Cardinale. Bologna: Il Mulino.

Dionigi, Ivano (a c. di)

2002

Di fronte ai classici. A colloquio con i Greci e i Latini. Milano: Rizzoli.

Eco, Umberto

2002

«Sulle spalle dei Giganti». En Di fronte ai classici. A colloquio con i Greci e i Latini, a c. di I. Dionigi. Milano: Rizzoli, pp. 115-139.

FAGGIN, Giuseppe

1964

«Scuola e cultura classica. Dobbiamo difendere la cultura umanistica?». En Atene e Roma. Rassegna trimestrale dell'Associazione Italiana di Cultura Classica. Nuova serie, VIII - Fasc. 1-2, pp. 1-18.

FLORES, Enrico

2001

Le due culture, l'umanistica e la scientifica, dall'antico al moderno e contemporaneo, «Vichiana», ser. IV, 3, pp. 135-147. 
Gentili, Bruno

2004

«Tra ricerca umanistica e ricerca scientifica». En Quaderni Urbinati di

Cultura Classica, N. 77, N. 2, pp. 143-157.

Gramsci, Antonio

1975 Quaderni del carcere, 12 (XXIX), 10, vols. I-IV. Ed. Critica dell'Istituto Gramsci, a c. di V. Gerratana. Torino: Editorial, p. 1544.

Hegel, Georg Wilhelm Friedrich

1930 Lezioni sulla storia della filosofia, I. Traducción de E. Codignola e G. Sanna. Firenze: La Nuova Italia.

1955 Lecciones sobre la historia de la filosofía I. Mexico D.F.: Fondo de Cultura Económica.

Jeauneau, Edouard

1969 «Nani sulle spalle di giganti». A cura di F. Lazzari. Napoli: Guida.

Montanari, Franco (a c. di)

2003

Rimuovere i classici. Cultura classica e società contemporanea. Torino: Einaudi scuola.

MÜLlER, Giuseppe

Prefazione. En SchenkL, Karl. Esercizi greci. Torino-Firenze: Loescher, pp. III-VII.

Narducci, Emanuele; Sergio Audano y Luca Fezzi (a c. di)

2005 «Aspetti della fortuna dell'antico nella cultura europea». Atti della prima giornata di studi, Sestri Levante, 26 marzo 2004, Pisa.

2008 "Aspetti della fortuna dell'antico nella cultura europea». Atti della quarta giornata di studi, Sestri Levante, 16 marzo 2007, Pisa. 


\section{CORRADO PETROCELLI}

Nussbaum, Martha

2011 Non per profitto. Perché le democrazie "hanno bisogno" della cultura umanistica. Bologna: Il Mulino.

SANGUineti, Edoardo

2002

"Classici e no". En Di fronte ai classici. A colloquio con $i$ Greci e $i$ Latini, a c. di I. Dionigi. Milano: Rizzoli, pp. 209-213.

SNELL, Bruno

1963

La cultura greca e le origini del pensiero europeo. Torino: Giulio Einaudi Editore.

Vacchina, Mariagrazia (a c. di)

1988 Attualità dell'antico. Aosta: Tipografia valdostana. 